\title{
Neuroprotective Effect of Physical Exercise on Neuronal Apoptosis Induced by Tramadol in Cerebral Cortex of Rats
}

\author{
Ashraf Albrakati $1, *$ (D) \\ 1 Department of Human Anatomy, College of Medicine, Taif University, Taif, Saudi Arabia \\ * Correspondence: mchakl@ hotmail.com;
}

Scopus Author ID 56674637400

Received: 4.05.2020; Revised: 2.05.2020; Accepted: 3.05.2020; Published: 7.06.2020

\begin{abstract}
Tramadol is a centrally acting analgesic agent with low affinity for opioid receptors, used for treating moderate to severe pain. Tramadol, like other opioids, induces neuronal apoptosis, which causes multiple neuronal impairments. The current study was conducted to evaluate the potential neuroprotective role of physical exercises on tramadol-induced neuronal apoptosis in the cerebral cortex of rats. Thirty adult male rats were divided into three groups $(n=10)$ as follow; the control group was gavaged with physiological saline $(0.9 \% \mathrm{NaCl})$; tramadol group was daily administered with tramadol (40 mg/kg) for 28 days, and physical exercise group was administered with the same dose as tramadol group, then rats were forced to run on the treadmill for $30 \mathrm{~min}$, once a day for 28 days. Tramadol induced histopathological changes in the form of neuroses degeneration and apoptosis. These findings were confirmed by immunohistochemical and blotting studies, which showed upregulation of p53 and downregulation of Bcl-2. In addition, malondialdehyde (MDA), myeloperoxidase (MPO), and nuclear factor kappa B (NF-kB) significantly increased following tramadol administration. At the same time, glutathione (GSH) and glutathione peroxidase (GPx) were decreased. In contrast, physical exercise was found to protect cortical neurons from degeneration and apoptosis produced by tramadol. This was evidenced by the downregulation of p53 and upregulating Bcl-2 expression and the improved changes in the oxidative stress biomarkers in rats. Physical exercise reduced the neuronal apoptosis and degeneration in the cerebral cortex following tramadol administration through suppressing oxidative stress.
\end{abstract}

Keywords: physical exercise; tramadol; apoptosis; oxidative stress; cerebral cortex.

(C) 2020 by the authors. This article is an open-access article distributed under the terms and conditions of the Creative Commons Attribution (CC BY) license (https://creativecommons.org/licenses/by/4.0/).

\section{Introduction}

The cerebrum is the most highly developed part of the brain that manages the essential and complex mental processes as language, thinking, perceiving, producing, and understanding [1]. The cerebral cortex is the largest brain region that controls learning, spatial memory, and other functions [2]. Tramadol is a synthetic codeine analog that is commonly used in treating severe to mild pains therapy and has an efficacy ranging from weak opioids to morphine [3]. Tramadol is categorized as a non-classical opioid combining the agonist activity of $\mu$-opioid receptors with blocking the activity of monoamine reuptake [4-8]. Although tramadol has a low incidence of side effects as respiratory depression and addiction compared to other opioids [9], several studies have linked between tramadol and the development of apoptosis in tissues of different organs as liver, kidney, and testis [10-14]. In addition, chronic tramadol 
administration causes histomorphological changes and impairs memory function in the cerebral cortex by activation of $\mu$-opioid receptors and enhancing the formation of red neurons and further apoptotic cascade $[15,16]$. Neuronal apoptosis produced by tramadol is thought to be involved in the development of several neurological deficits. It was reported that chronic administration of tramadol had been associated with reactive oxygen species (ROS) production and the development of oxidative stress [16]. ROS is known to attack DNA, proteins, and lipids, and enhance neuronal apoptosis, and further produce sever cellular dysfunction [18-20].

The protective role of the natural products and/or supplements on neuronal apoptosis induced by tramadol in cerebral cortex showed a decrease in the neuronal apoptosis in rats cerebral cortex, but not fully cured [21-23]. Furthermore, influences of withdrawal were slightly associated with oxidative stress inhibition and improvement of histological abnormalities in the brain tissue [16,24]. Interestingly, treadmill exercise showed a neuroprotective effect in the schizophrenic model through inhibiting apoptotic cascade. Also, physical exercise decreased the number of hippocampal apoptotic cells in ischemic rats [25,26]. Moreover, Physical exercise has been found to protect brain tissue against neuronal degeneration through inhibiting oxidative stress [26]. Hence, this study was designed to evaluate the potential neuroprotective role of physical exercise on cortical apoptosis and oxidative stress induced by tramadol.

\section{Materials and Methods}

\subsection{Experimental animals.}

Thirty male Wistar albino rats (200-250 g; 10 weeks old) were used in this study. Animals were obtained from the animal house of King Abdulaziz University, Jeddah, Saudi Arabia. Animals were fed on a standard laboratory food and kept in specially designed cages and kept for acclimatization under standard conditions for at least one week.

\subsection{Animals groups and doses of the treatment.}

Tramadol hydrochloride was purchased from (ADWIA, 10th of Ramadan City, Egypt), each ampoule contains $20 \mathrm{mg}$. Animals were divided into three groups (10 each) as follows: control group, tramadol group, and physical exercise group. Rats in the control group were administered with physiological saline $(0.9 \% \mathrm{NaCl})$ for four weeks. Rats in the tramadol group were treated with tramadol (40 mg/kg) according to El-Ghawet [27] for four weeks. Rats in the physical exercise group were administered with the same dose as the group of tramadol. Then rats were forced to run for $30 \mathrm{~min}$ once a day on the treadmill. All animal groups were sacrificed by the end of the fourth week. The experiment was conducted at the laboratories of the Faulty of Medicine, Taif University, Taif, Saudi Arabia.

\subsection{Exercise protocol}

The treated-rats in the physical exercise group were forced to run on a motorized treadmill for 30 min once a day for 28 days. The exercise load consisted of running at a speed of 2 meters/min for the first $5 \mathrm{~min}, 5$ meters/min for the next $5 \mathrm{~min}$, and 8 meters/min for 20 min, at room temperature. Animals in the physical exercise group were sacrificed after their final exercise session [25]. 


\subsection{Histopathological and immunohistochemical examinations.}

After the animals were sacrificed, brains were obtained from the skulls. For histopathological and Immunohistochemical studies, the cerebral cortex area was immediately separated from the brain. The cerebral cortex was fixed in $10 \%$ neutral buffered formalin. Following fixation, specimens were dehydrated, embedded, and then cut at $8 \mu \mathrm{m}$ thickness using Leica microtome (Leica RM 2025; Nassloch, Germany). Then, sections of the cerebral cortex were mounted on the clean slides and left-over night to dry. For histological examination, sections of the cerebral cortex were stained with Ehrlich Hematoxylin and Eosin [28], while immunohistochemical sections were placed on positive coated slides and then stained with anti-p53 and anti-Bcl-2 according to the method of Palermo et al. [29]. The slides were incubated with primary rabbit antibodies, anti-p53 (1:500, Biotechnology, Santa Cruz, USA) and anti-Bcl-2 (1:300, Biotechnology, Santa Cruz, USA) for 2 hours. Consequently, the slides were re-incubated with goat anti-rabbit (dilution 1:1000, Biotechnology, Santa Cruz, USA) as a secondary antibody for 1 hour at room temperature. Sections of the cerebral cortex stained with anti-p53 appear brown-colored nuclei of the neurons, indicating favorable neuronal apoptosis. Perinuclear membrane peri-Bcl-2 stained cerebral cortex segments emerged in brown-colored neurons, indicating favorable neuronal apoptosis.

\subsection{Counting of the apoptotic cells in the cortical tissue.}

The number of apoptotic cells in rats' cerebral cortex were counted using an Olympus microscope with a $\times 40$ objective lens using rectangular grids placed randomly in the investigated areas. Morphometrical methods were applied to count apoptotic cells per unit area in the cerebral cortex. Apoptotic cells were recognized in the histology and were identified on the basis of apoptotic cell morphological criteria as; brightly stain with scanty eosinophilic cytoplasm, nuclear pyknosis, and forming of apoptotic membrane-bounded bleb [30-32]. The mean number of apoptotic cells per unit area in different regions of the cerebral cortex were calculated according to the method of Baghishani et al. [33].

2.6. Counting of the stained neurons with anti-p53 and anti-Bcl-2 in the cerebral cortex of rats.

The stained neurons of cerebral cortex tissues with anti-p53 and anti-Bcl-2 were counted using an Olympus microscope with a $\times 40$ objective lens using rectangular grids placed randomly in investigated areas and away from lesions. Examination of the immunohistochemical sections showed nuclei of the neurons with brown color (for p53) and perinuclear membrane of the neurons with brown color (for Bcl-2), which considered as a positive marker of apoptotic neuronal cells. Semi-quantification analysis of the apoptotic index (AI) was determined by counting a total of at least 1000 cells per slide subdivided in 10 fields chosen randomly at $\times 400$ magnification. $\mathrm{AI} \%=$ [number of positive cells/total number of calculated cells] $\times 100$, which represented the percentage of positive cells in 1000 cells [32].

\subsection{Western blot.}

According to the method described by Liu et al. [34], protein was extracted from the cerebral cortex using ice-cold RIPA buffer in the presence of protease and phosphatase inhibitors. Samples were homogenized by sonication and centrifuged at $14,000 \mathrm{rpm}$ at $4^{\circ} \mathrm{C}$ for $30 \mathrm{~min}$. Supernatants were collected and stored at $-80^{\circ} \mathrm{C}$. Standard Laemmli buffer was added, and samples were boiled for $5 \mathrm{~min}$. Equal amounts of protein were resolved in gradient 10 
$20 \%$ SDS-PAGE gel and transferred to a polyvinylidene difluoride (PVDF) membrane overnight. Membranes were blocked with 5\% non-fat milk in Tris buffer saline, $0.1 \%$ Tween (TTBS) for 1 hour. Membranes were incubated at $4{ }^{\circ} \mathrm{C}$ overnight with primary rabbit antibodies, anti-p53, anti-Bcl-2, and anti- $\beta$-actin. Goat anti-rabbit HRP-conjugated immunoglobulins were used as the secondary antibody for 1 hour at room temperature. After stripping, membranes were re-probed with mouse monoclonal antibody $\beta$-actin as an internal control for loading and transfer of proteins. Band detection was performed using the enhanced chemiluminescence (ECL) detection kit (Bio-Rad), and the light was captured using auto-radiographic Hyperfilm in a hyper-cassette. Hyperfilm was developed and fixed, and the image was scanned so that bands can be sized against the pre-stained rainbow ladder, and the obtained bands were quantified using a scanning densitometer (model 670, Bio-Rad). Western blots of p53 and Bcl2 were quantified according to the method described by Taylor C. et al. [35]. The results were normalized to $\beta$-actin and presented as a percent of control.

\subsection{Oxidative stress and inflammatory markers in the cortical tissue.}

At the end of the experiment, rats of all experimental groups were sacrificed under anesthesia, and samples were collected. Frontal parts from the cerebral cortex were homogenized in cold phosphate-buffered saline (PBS), centrifuged, and the clear homogenate was collected for biochemical assays. The formed supernatant was stored at $-80^{\circ} \mathrm{C}$ for later use in biochemical measurements. The brain protein content was measured according to the Lowry protocol [36]. The lipid peroxidation marker MDA estimation was determined according to the protocol of Ohkawa et al. [37]. Levels of GSH was estimated according to the protocol of Elman [38]. The activity of GPx was measured using the method described by Paglia and Valentine [39]. Myeloperoxidase (MPO) activity and nuclear factor kappa B (NF-кB) content in the brain homogenates were assessed based on the method of Bradly et al. [40] and Hussein et al. [41], respectively.

\subsection{Ethical considerations.}

All experiment protocols, including the use of animals, were approved by the Committee of Research Ethics for Laboratory Animal Care, Anatomy Department, School of Medicine, Taif University (approval no, 40-36-0191).

\subsection{Statistical analysis.}

All analyses were carried out using SPSS version17. Data collected from all techniques used in this study were presented as mean \pm SEM. Data were analyzed using statistical t-tests. $* \mathrm{P}<0.05, * * \mathrm{P}<0.01$ and $* * * \mathrm{P}<0.001$.

\section{Results and Discussion}

\subsection{Histology of the cerebral cortex.}

Histological examination of the control group showed that the cerebral cortex is consist of groups of nerve cells arranged in six layers from outside inwards: I; outer molecular layer, II; outer granular layer, III; outer pyramidal layer, IV; inner granular layer, V; inner pyramidal and VI; polymorphic layers (figure $1 \mathrm{~A}$ ). Whereas, the outer molecular layer showed comparatively consists of fibrous tissue with few neurons and neuroglia cells. Both inner and 
outer pyramidal layers consist of large and medium-sized pyramidal cells, respectively. The inner and outer granular layers showed to consist of a stellate-shaped nerve with large round nuclei (figure $1 \mathrm{~B}$ ) while polymorphic layer showed to consist of a variety of nerve cells in different sizes.
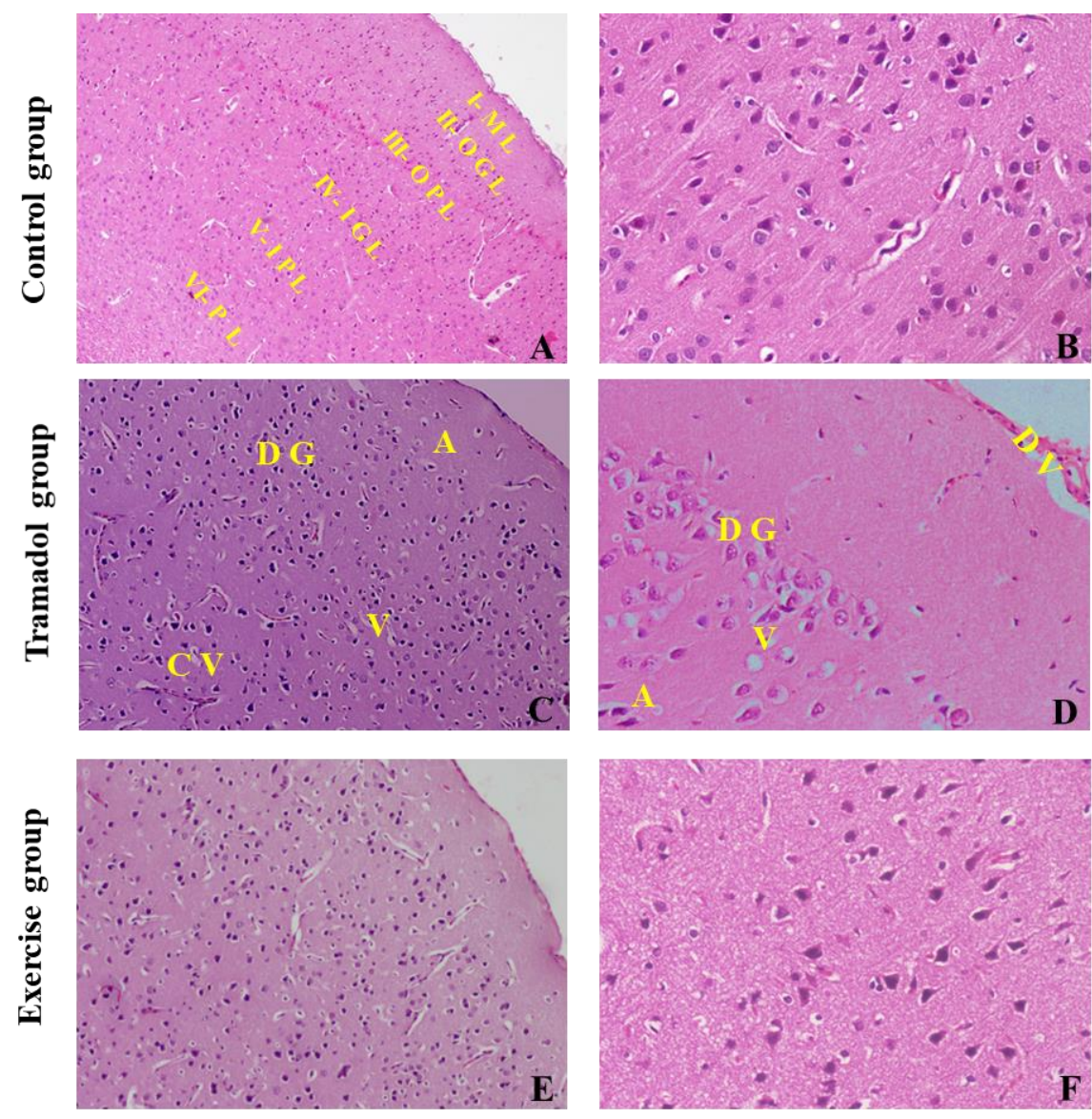

G

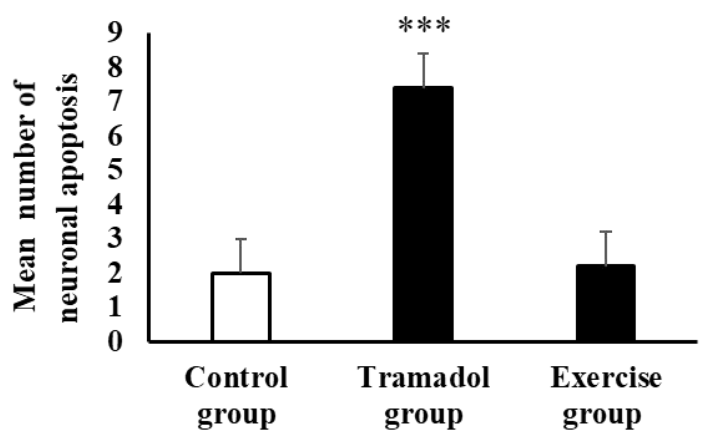

Figure 1. Effect of physical exercise on cortical histological alterations following tramadol administration. (A and $\mathrm{B}$ ) control group, (C and D) tramadol administered group, and (E and F) tramadol and physical exercised group. G; shows the mean number of the apoptotic cells of the control group, tramadol group, and physical exercise group. A, C, and E x200. B, D, and F x400. Data are expressed as Mean \pm SEM, $\mathrm{n}=10$. *P $<0.05$, **P $<0.01$ and $* * * \mathrm{P}<0.001$.

\subsection{Cortical histopathological changes following tramadol administration.}

Light microscopic examination of the tramadol treated group showed severe multifocal histological changes in all cortical layers as compared to the control group. Disorganization of cortical layers with degenerated neurocytes, vacuolation of neuropil, vacuolated foci with cellular loss and intense eosinophilic staining of neuropil. In this group, congested and dilated blood vessels were also shown (figure $1 \mathrm{C}$ ). Degenerative changes of apoptotic neurons were 
characterized by irregular nuclei in some neuronal cells with brightly stained scanty eosinophilic cytoplasm, darkly stained nuclei, and perineural retraction spaces. The pyramidal cells in both pyramidal layers were appeared irregular in shape with deeply stained nuclei. Most cells of granule layers were appeared with faintly stained and loss of their nucleoli. However, apoptotic cells, degenerative and necrotic cells were more marked in the treated rats (figure 1 D).

\subsection{Effects of physical exercise on the cortical histopathological changes following tramadol administration.}

Microscopic examination of rats' cerebral cortex in the physical exercise group showed the return of cortical tissues nearly to the typical histological structure as evidenced by the decreased number of apoptotic cells and remarkable regression of the degenerative changes and decreased perineuronal haloes that induced by tramadol (figure $1 \mathrm{E}$ ). However, multiple pyramidal cells and granular cells appeared normal; but, some of the pyramidal cells were still affected in between the normal granule cells and appeared shrunken with darkly stained nuclei. Granule cells that lost their nuclei appeared faintly stained. Neuroglial cells were also nearly similar to those neuroglial cells in the control group. However, few of these cells appeared more darkly stained, and the neuropil was still vacuolated in some areas (figure $1 \mathrm{~F}$ ).

3.4. Effects of physical exercise on apoptotic cells count in the cortical tissue following tramadol administration.

Apoptotic cell analytical score showed a few numbers of apoptotic cells in the control group. While the average number of apoptotic neurons in the tramadol group improved considerably $(\mathrm{P}<0.001)$ relative to the control group. In contrast, the mean number of apoptotic cells in the physical exercise group were significantly decreased $(\mathrm{p}<0.001)$ when compared to the tramadol group and non-significant as compared to the control group (figure $1 \mathrm{G}$ ).

\subsection{Effects of physical exercise on $p 53$ and Bcl-2 expression following tramadol administration.}

Immunohistochemical results showed that few numbers of neurons were negatively stained with anti-p53 (figure $2 \mathrm{~A}$ ) and positively with anti-Bcl-2 in the control group (figure 2 B). Tramadol group showed a large number of neurons that stained positively with anti-p53 (figure 2 C) and weakly stained with anti-Bcl-2 (figure $2 \mathrm{D}$ ). In contrast, the physical exercised group showed a few numbers of the neurons stained positively with anti-p53 (figure $2 \mathrm{E}$ ) and a few numbers stained with anti-Bcl-2 (figure $2 \mathrm{~F}$ ). Analysis of stained neurons with anti-p53 and anti-Bcl-2 as an apoptotic marker of the neurocytes in the cerebral cortex of rats in tramadol group showed a significant increase $(\mathrm{P}<0.001)$ of p53 (figure $2 \mathrm{G}$ ) along with a significant decrease $(\mathrm{P}<0.001)$ of $\mathrm{Bcl}-2$ as compared to control group. In contrast, the exercised group exhibited neuroprotective effect through decrease negative staining with anti-p53 (figure $2 \mathrm{H}$ ) and increased positive staining with anti-Bcl-2 (figure $2 \mathrm{G}$ ) following tramadol administration as compared to the control group, but still partially different when compared to tramadol group.

\subsection{Effect of physical exercise on p53 and Bcl-2 protein levels.}

In this study, Western blot was used to study the impact of treadmill exercise on the expression of p53 and Bcl-2 following tramadol administration in the cerebral cortex of rats. 
Western blot of the control group showed that Bcl-2 protein was constitutively expressed in the cerebral cortex of rats, whereas p53 protein was weakly expressed in the same group (figure $3 \mathrm{~A})$.
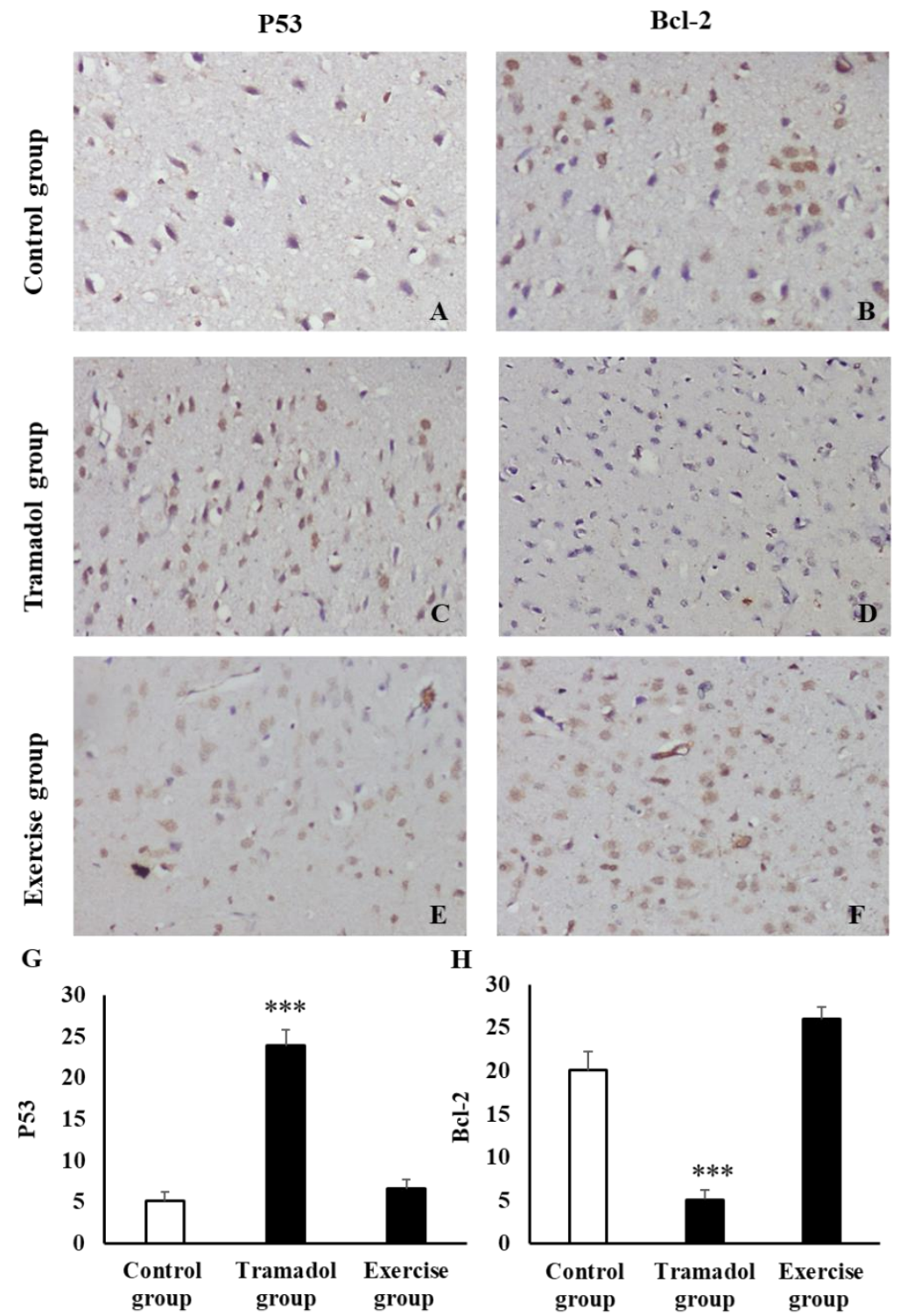

Figure 2. Expression of p53 and Bcl-2 in control, tramadol, and physical exercised groups using immunohistochemistry. (A and D) Control, (B and E) tramadol, and (C and F) tramadol and physical exercised group. $\mathrm{G}$ and $\mathrm{H}$ show changes in the apoptotic index of the neurocytes of the cerebral cortex tissue stained with anti-p53 and anti-Bcl-2 in the control group, tramadol group, and physical exercise group. A, B, C, D, E, and F $\mathrm{x} 400$. Data are expressed as Mean $\pm \mathrm{SEM}, \mathrm{n}=10 . * \mathrm{P}<0.05, * * \mathrm{P}<0.01$ and $* * * \mathrm{P}<0.001$.

Meanwhile, the tramadol treated group showed a significant increase $(\mathrm{P}<0.001)$ in $\mathrm{p} 53$ protein expression (figure $3 \mathrm{~B}$ ) and a significant decrease $(\mathrm{P}<0.001)$ in $\mathrm{Bcl}-2$ protein expression as compared with the control group (figure $3 \mathrm{C}$ ). The exercise group showed that the expression proteins levels of p53 restored to the control level as compared with the tramadol group, along with increased Bcl-2 protein expression as compared with the control group as presented in figures $3 \mathrm{~B}$ and $\mathrm{C}$, respectively.

\subsection{Effect of physical exercise on the oxidative status following tramadol administration.}

The tramadol-intoxicated group showed a significant increase in MDA level $(\mathrm{P}<0.001)$ as compared to control levels (figure $4 \mathrm{~A}$ ), whereas a significant decrease in GSH and GPx 
activity $(\mathrm{P}<0.01)$ was observed as shown in figures. $4 \mathrm{C}$ and $\mathrm{D}$, respectively. Notably, physical exercise was found to decrease MDA (figure 4 A) and increase GSH content and GPx activity when compared against the tramadol-intoxicated group, as shown in figures. C and D, respectively.

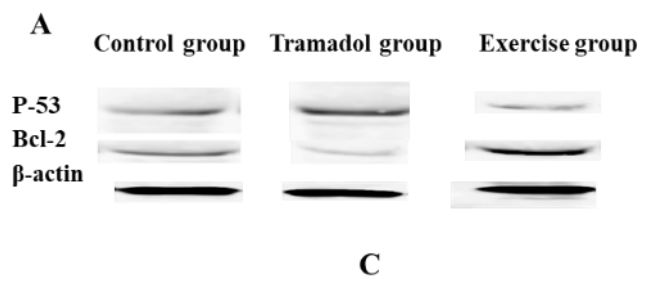

B
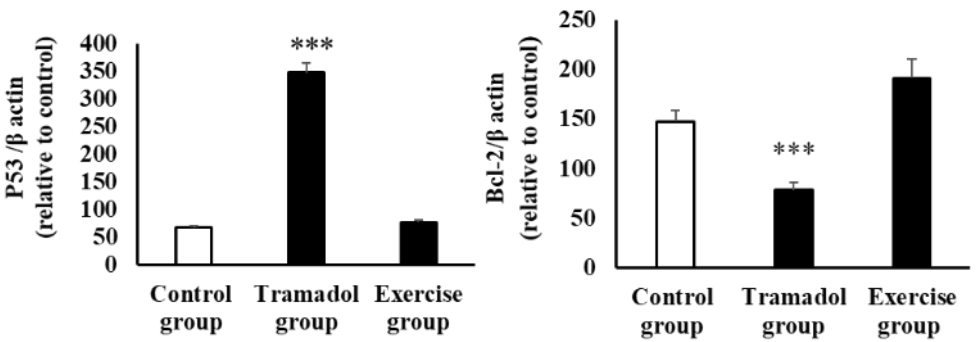

Figure 3. Representative blots of $\mathrm{p} 53, \mathrm{Bcl}-2$, and $\beta$-actin in the control group, tramadol group, and physical exercise group (A). B and C show Western blot analysis of Bcl-2 protein and P53 protein in the cerebral cortex of rats in the control group, tramadol group, and physical exercise group. Data are expressed as Mean \pm SEM, $\mathrm{n}=10 . * \mathrm{P}<0.05, * * \mathrm{P}<0.01$ and $* * * \mathrm{P}<0.001$.

\subsection{Effect of physical exercise on MPO activity and $N F-\kappa B$ following tramadol administration.}

Rats treated with tramadol showed a significant elevation in MPO activity $(\mathrm{P}<0.01)$ and $\mathrm{NF}-\kappa \mathrm{B}$ content $(\mathrm{P}<0.01)$ as compared to control levels, whereas a significant decrease was observed in physical exercise exposed group following tramadol treatment when compared against the tramadol-intoxicated group as shown in figures. $4 \mathrm{D}$ and $\mathrm{E}$, respectively
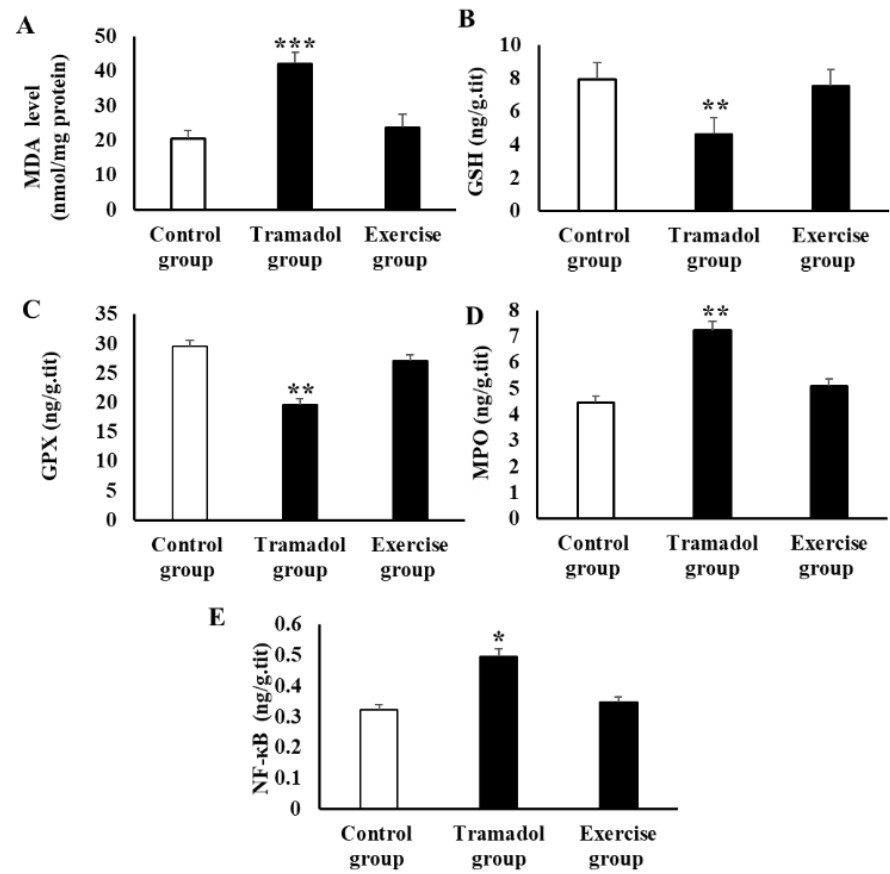

Figure 4. Shows oxidant and antioxidant markers of rat cerebral cortex tissue of the control group, tramadol group, and physical exercise group. Results are presented as mean $\pm \mathrm{SEM}, \mathrm{n}=10$. $* \mathrm{P}<0.05$, **P $<0.01$ and $* * * \mathrm{P}<0.001$. 


\subsection{Discussion.}

The cerebral cortex is the most important region in the brain, controlling several neuronal functions like learning and spatial memory [2]. It is known that excessive tramadol administration induces several histopathological changes in the brain tissues, including apoptosis, which triggers the development of numerous neurological impairments [42]. The neuroprotective effect of natural products and against apoptosis induced by tramadol in the brain tissues have been discussed in previous studies [21,24]. Here, we investigated the potential protective role of physical exercise on neuronal apoptosis in the cortical tissue following tramadol administration. It has been reported that chronic tramadol administration was found to be associated with structural changes in pyramidal cells with faintly stained cytoplasm and nuclei, as well as increased apoptotic cells in the cortical tissue [16]. In addition, tramadol enhanced degenerative changes in the neural cells and changed the thickness of the cerebral cortex and hippocampus of rats [43]. In the current study, tramadol administration increased the apoptotic cells in the cortical tissue. These results are partially in agreement with a previous report [21], the authors reported that administration of different tramadol doses caused a significant increase in the shrunken pyramidal cells in rat cerebral cortex [21].

The histopathological results were confirmed by IHC studies that showed a large number of apoptotic neurons in the tramadol treated group and also showed a positive reaction for $\mathrm{p} 53$. Few apoptotic neurons have a positive reaction for Bcl-2 in the cerebral cortex. These findings are in line with Shaibah et al. [44], who recorded a significant increase in p53 expression accompanied by a decrease in $\mathrm{Bcl}-2$ expression in the cortical tissue following tramadol administration.

In a step to evaluate the protective role of the physical exercise on neuronal apoptosis induced by tramadol in the cortical tissue, rats were forced to run on the treadmill for $30 \mathrm{~min}$ daily for 4 weeks. Our findings revealed a significant improvement in the apoptotic reactions and histological alterations in the physical exercised group. However, some pyramidal cells in the physical exercise group were still affected and appeared shrunken with darkly stained nuclei. Increasing blood supply into the brain tissue during the physical exercise was found to increase oxygen and glucose supply into the brain tissue, which helps to protect the tissue and the cells from apoptosis [45]. Also, regular physical exercise protected the central nervous system (CNS) against aging diseases and helped to recovery after CNS injury [46]. In this regard, it has been reported that the physical exercise ameliorates complex partial seizures induced by tramadol through decreasing the neuronal apoptosis in the hippocampus [47]. To further confirm the development of neuronal apoptosis in the cerebral cortex following tramadol treatment, the pro-apoptotic p53 and anti-apoptotic Bcl-2 proteins were examined using IHC and blotting methods [48]. Tramadol administration was found to be associated with the upregulation of p53 and the downregulation of Bcl-2. These results confirm the histopathological result following tramadol treatment, which showed apoptotic cells in several layers of the cerebral cortex. These results are in line with Shaibah et al. [44], who reported that the histopathological deformations were coupled with p53 upregulation. Additionally, Khodeary et al. [49] demonstrated that the administration of tramadol with high and low doses caused alteration in p53 and Bcl-2 apoptotic pathway homeostasis in cerebral cortex and hippocampus. It has been reported that chronic use of opiates was associated with a significant increase of pro-apoptotic Fas receptor and decrease expression of $\mathrm{Bcl}-2$ in rats cerebral cortex tissue [50]. Several studies have shown the neuroprotective role of the physical exercise on cell 
apoptosis in the brain tissue in different models [25,51], and it was found to regulate the expression of pro-apoptotic, p53 and anti-apoptotic, Bcl-2 proteins [25,34]. In the current study, physical exercise was found to decrease the expression of p53 protein and upregulated Bcl-2 expression in the cortical tissue in response to tramadol administration. It has been shown that physical exercise inhibited neuronal apoptosis via inhibiting NMDA receptor expression in schizophrenic mice [25]. Moreover, [51] reported that the force ischemic-Gerbils to run on a treadmill for thirty min per a day ameliorated nervous system complications such as the shortterm memory deficits, through inhibiting neuronal apoptosis, and thus improve the cortical tissue.

Several studies have linked between neuronal apoptosis and oxidative stress in brain tissue following tramadol administration [24,52,53]. Apoptosis can be initiated responses to several agents as DNA damage or opioids, which lead to the activation of oxidative stress [31]. In another study, it has been reported that the chronic administration of tramadol was found to enhance ROS production in brain tissues, which attack DNA, lipids, and other macromolecules [16]. In this study, the biochemical results showed an increase in MDA, MPO, and NF- $\kappa B$ following tramadol administration, while GSH and GPx were decreased. Awadalla et al. [43] reported that the tramadol significantly increased MDA level and decreased GSH, SOD, and CAT levels in cortex and hippocampus tissues of rats. The elevated MDA could be due to the overproduction of ROS [54]. Hussein et al. [24] stated that the treatment with both the therapeutic and overdoses of tramadol for four weeks induced a significant decline in the level GSH and the activity of GPx, and an increase in MDA and MPO activity in the brain tissue. Ismail et al. [52] reported that tramadol inhibited GPx activity through the interaction with the transition metal in the GPx enzyme. It has been reported that the neuronal damage in the brain tissue of mice was oxidative stress-dependent, and suppressor activity of MPO conserves neuronal function via inhibits oxidative stress and then cell apoptosis [55].

The biochemical results showed a significant increase in NF- $\kappa \mathrm{B}$ in cortical tissue following tramadol administration. NF- $\mathrm{kB}$ is one of the family transcription factors which regulate the inflammation in response to injury and infection. NF-kB is known to enhance the production of cytokines, inflammatory mediators, and apoptosis. NF- $\mathrm{KB}$ can be stimulated in response to several factors, including ROS, which disturbs DNA transcription and enhances cytokine production, and cell apoptosis $[17,56]$. It has been reported that the activation of NF$\kappa \mathrm{B}$ has been associated with the development of inflammation and oxidative stress following tramadol administration in rats' brain tissue [24]. Mohamed et al. [22] reported that tramadolinduced neurotoxicity depends on oxidative stress, inflammation, and apoptosis, which is associated with the elevation of lipid peroxidation and upregulated inflammation markers in the cerebrum tissue.

Regular exercise inhibits lipid peroxidation and enhances antioxidant enzymes [57]. Upregulating the activity of antioxidant molecules is expected to inhibit neurodegenerative and cell apoptosis [26]. Biochemical results of the physical exercise group showed a significant increase in GSH content, GPx activity, and decreased MPO activity in the cortical tissue, coupled with a significant deactivation of NF- $\kappa \mathrm{B}$. The results of this study reflect the neuroprotective role of physical exercise against tramadol-induce oxidative stress, inflammation, and apoptosis in the cortical tissue. Ferrer et al. [57] stated that the physical exercise activates catalase as a part of the antioxidant defenses to avoid neutrophil-induced oxidative stress. Besides, activation of catalase leads to the inhibition of cytochrome c release, 
which prevents the formation of caspase activation complexes and thus inhibiting the release of caspase-independent proteins.

\section{Conclusions}

Collectively, tramadol was found to induce histological changes in the cortical structure, enhanced apoptotic pathway through upregulating the expression of $\mathrm{p} 53$, and downregulating Bcl-2 expression. Also, tramadol disturbs the oxidative status via enhancing the oxidation of lipids, decreasing glutathione, and glutathione peroxidase. In addition, tramadol enhances inflammatory response as indicated by the elevated MPO and NF- $\kappa$ B levels. However, physical exercise reversed these alterations to be near the control values, reflecting its neuroprotective effect against tramadol-induced neurotoxicity.

\section{Funding}

This research received no external funding.

\section{Acknowledgments}

This research received no acknowledgment.

\section{Conflicts of Interest}

The authors declare no conflict of interest.

\section{References}

1. Angevine, J.B.; Cotman, C.W. Principles of neuroanatomy. Oxford University Press, USA, 1981.

2. Pandya, D.; Petrides, M.; Cipolloni, P.B. Cerebral cortex: architecture, connections, and the dual origin concept. Oxford University Press: 2015, https://doi.org/10.1093/med/9780195385151.001.0001.

3. Eggers, K.A.; Power, I. Tramadol. British journal of anaesthesia 1995, 74, 247-249, https://doi.org/10.1093/bja/74.3.247.

4. Vadivelu, N.; Mitra, S.; Narayan, D. Recent advances in postoperative pain management. The Yale journal of biology and medicine 2010, 83, 11-25.

5. Power, I. An update on analgesics. British journal of anaesthesia 2011, 107, 19-24, https://doi.org/10.1093/bja/aer126.

6. Pergolizzi, J.; Alegre, C.; Blake, D.; Alen, J.C.; Caporali, R.; Casser, H.R.; Correa-Illanes, G.; Fernandes, P.; Galilea, E.; Jany, R.; Jones, A.; Mejjad, O.; Morovic-Vergles, J.; Oteo Álvaro, Á.; Radrigán Araya, F.J.; Simões, M.E.C.; Uomo, G. Current considerations for the treatment of severe chronic pain: the potential for tapentadol. Pain practice: the official journal of World Institute of Pain 2012, 12, 290-306, https://doi.org/10.1111/j.1533-2500.2011.00487.x.

7. Singh, D.R.; Nag, K.; Shetti, A.N.; Krishnaveni, N. Tapentadol hydrochloride: A novel analgesic. Saudi journal of anaesthesia 2013, 7, 322-326, https://doi.org/10.4103/1658-354x.115319.

8. Tzschentke, T.M.; Christoph, T.; Kogel, B.Y. The mu-opioid receptor agonist/noradrenaline reuptake inhibition (MOR-NRI) concept in analgesia: the case of tapentadol. CNS drugs 2014, 28, 319-329, https://doi.org/10.1007/s40263-014-0151-9.

9. Yuan, J.; Yankner, B.A. Apoptosis in the nervous system. Nature 2000, 407, 802-809, https://doi.org/10.1038/35037739.

10. Yin, M.; Jiang, N.; Guo, L.; Ni, Z.; Al-Brakati, A.Y.; Othman, M.S.; Moneim, A.E.A.; Kassab, R.B. Oleuropein suppresses oxidative, inflammatory, and apoptotic responses following glycerol-induced acute kidney injury in rats. Life sciences 2019, 232, https://doi.org/10.1016/j.lfs.2019.116634.

11. Nash, S.; Rahman, M.S. Short-term heat stress impairs testicular functions in the American oyster, Crassostrea virginica: Molecular mechanisms and induction of oxidative stress and apoptosis in spermatogenic cells. 2019, 86, 1444-1458, https://doi.org/10.1002/mrd.23268.

12. Al-Brakati, A.Y.; Kassab, R.B.; Lokman, M.S.; Elmahallawy, E.K.; Amin, H.K.; Moneim, A.E.A. Soursop fruit extract mitigates scopolamine-induced amnesia and oxidative stress via activating cholinergic and Nrf2/HO-1 pathways. Metabolic Brain Disease 2019, 34, 1-12, https://doi.org/10.1007/s11011-019-004072 . 
13. Al-Brakati, A.Y.; Fouda, M.S.; Tharwat, A.M.; Elmahallawy, E.K.; Kassab, R.B.; Moneim, A.E.A. The protective efficacy of soursop fruit extract against hepatic injury associated with acetaminophen exposure is mediated through antioxidant, anti-inflammatory, and anti-apoptotic activities. Environmental Science and Pollution Research 2019, 26, 13539-13550, https://doi.org/10.1007/s11356-019-04935-3.

14. Albarakai, A.Y.; Alsbery, H.M.A.E. Evaluation of the Hepatoprotective Efficacy of Moringa oleifera on Tramal-Induced Liver Toxicity in Animal Modules. Research journal of pharmaceutical biological and chemical sciences 2016, 7, 1494-1501.

15. Hosseini-Sharifabad, A.; Rabbani, M.; Sharifzadeh, M.; Bagheri, N. Acute and chronic tramadol administration impair spatial memory in rat. Research in pharmaceutical sciences 2016, 11, 49-57.

16. Ghoneim, F.M.; Khalaf, H.A.; Elsamanoudy, A.Z.; Helaly, A.N. Effect of chronic usage of tramadol on motor cerebral cortex and testicular tissues of adult male albino rats and the effect of its withdrawal: histological, immunohistochemical and biochemical study. International journal of clinical and experimental pathology 2014, 7, 7323-7341.

17. Uttara, B.; Singh, A.V.; Zamboni, P.; Mahajan, R.T. Oxidative stress and neurodegenerative diseases: a review of upstream and downstream antioxidant therapeutic options. Curr Neuropharmacol 2009, 7, 65-74, https://doi.org/10.2174/157015909787602823.

18. Sharath Chandra, S.; Sukumaran, S. Magnesium chloride impairs physio-biochemical and neurochemical responses in cirrhinusmrigala (Hamilton, 1822) upon short term exposure. Biointerface Research in Applied Chemistry 2020, 10, 4934-4938.

19. Al-Brakati, A. Protective effect of aged garlic extracts against hepatotoxicity induced by ethephon in Wistar albino rat. Environmental science and pollution research international 2020, 27, 6139-6147, https://doi.org/10.1007/s11356-019-07148-w.

20. Omar, N.M. Nigella sativa oil alleviates ultrastructural alterations induced by tramadol in rat motor cerebral cortex. Journal of Microscopy and Ultrastructure 2016, 4, 76-84, https://doi.org/10.1016/j.jmau.2015.12.001.

21. Mohamed, H.M.; Mahmoud, A.M. Chronic exposure to the opioid tramadol induces oxidative damage, inflammation and apoptosis, and alters cerebral monoamine neurotransmitters in rats. Biomedicine \& pharmacotherapy $=$ Biomedecine \& pharmacotherapie 2019, 110, 239-247, https://doi.org/10.1016/j.biopha.2018.11.141.

22. Hassan, A.; Ullah, H.; Israr, M. The antioxidant activity and phytochemical analysis of medicinal plant Veronica biloba. Letters in Applied NanoBioScience 2019, 8, 732-738, https://doi.org/10.33263/LIANBS84.732738.

23. Hussein, S.A. Effect of tramadol drug on some biochemical and immunological parameters in albino male rats; evaluation of possible reversal following its withdrawal. 2017, 33, 418-429, https://doi.org/10.21608/BVMJ.2017.30589.

24. Chung, J.W.; Seo, J.H.; Baek, S.B.; Kim, C.J.; Kim, T.W. Treadmill exercise inhibits hippocampal apoptosis through enhancing N-methyl-D-aspartate receptor expression in the MK-801-induced schizophrenic mice. Journal of exercise rehabilitation 2014, 10, 218-224, https://doi.org/10.12965/jer.140144.

25. Mourão, F.A.G.; Leite, H.R.; de Carvalho, L.E.D.; Ferreira e Vieira, T.H.; Pinto, M.C.X.; de Castro Medeiros, D.; Andrade, I.L.L.; Gonçalves, D.F.; Pereira, G.S.; Dutra Moraes, M.F. Neuroprotective effect of exercise in rat hippocampal slices submitted to in vitro ischemia is promoted by decrease of glutamate release and pro-apoptotic markers. Journal of neurochemistry 2014, 131, 65-73, https://doi.org/10.1111/jnc. 12786.

26. El-Ghawet, H.A. Effects of tramadol on the reproductive function of wistar albino rats. Eur J Exp Biol 2015, 5, 56-64.

27. Bancroft, J.D.; Gamble, M. Theory and practice of histological techniques. Elsevier health sciences: US, 2008.

28. Palermo, I.; Arcidiacono, G.; Barone, N.; Italia, F.; Arizzi, M.; Spera, G.; Bartoloni, G.; Di Mercurio, S.; Vicari, E.; Calogero, A.E. Normal expression of isoforms activating cyclic adenosine monophosphate responsive element modulator in patients with spermatid maturation arrest. Fertility and sterility 2004, 82 Suppl 3, 1072-1076, https://doi.org/10.1016/j.fertnstert.2004.05.077.

29. Burke, C.; Sinclair, K.; Cowin, G.; Rose, S.; Pat, B.; Gobe, G.; Colditz, P. Intrauterine growth restriction due to uteroplacental vascular insufficiency leads to increased hypoxia-induced cerebral apoptosis in newborn piglets. Brain research 2006, 1098, 19-25, https://doi.org/10.1016/j.brainres.2006.04.129.

30. Edwards, A.D.; Yue, X.; Cox, P.; Hope, P.L.; Azzopardi, D.V.; Squier, M.V.; Mehmet, H. Apoptosis in the brains of infants suffering intrauterine cerebral injury. Pediatric research 1997, 42, 684-689, https://doi.org/10.1203/00006450-199711000-00022.

31. Xu, C.; Shu, W.Q.; Qiu, Z.Q.; Chen, J.A.; Zhao, Q.; Cao, J. Protective effects of green tea polyphenols against subacute hepatotoxicity induced by microcystin-LR in mice. Environmental toxicology and pharmacology 2007, 24, 140-148, https://doi.org/10.1016/j.etap.2007.04.004.

32. Baghishani, F.; Mohammadipour, A.; Hosseinzadeh, H.; Hosseini, M.; Ebrahimzadeh-bideskan, A. The effects of tramadol administration on hippocampal cell apoptosis, learning and memory in adult rats and 
neuroprotective effects of crocin. Metabolic brain disease 2018, 33, 907-916, 1-10, https://doi.org/10.1007/s11011-018-0194-6.

33. Liu, G.; Keeler, B.E.; Zhukareva, V.; Houle, J.D. Cycling exercise affects the expression of apoptosisassociated microRNAs after spinal cord injury in rats. Experimental neurology 2010, 226, 200-206, https://doi.org/10.1016/j.expneurol.2010.08.032.

34. Taylor, S.C.; Berkelman, T.; Yadav, G.; Hammond, M. A defined methodology for reliable quantification of Western blot data. Molecular biotechnology 2013, 55, 217-226, https://doi.org/10.1007/s12033-0139672-6.

35. Lowry, O.H.; Rosebrough, N.J.; Farr, A.L.; Randall, R.J. Protein measurement with the Folin phenol reagent. The Journal of biological chemistry 1951, 193, 265-275.

36. Ohkawa, H.; Ohishi, N.; Yagi, K. Assay for lipid peroxides in animal tissues by thiobarbituric acid reaction. Analytical biochemistry 1979, 95, 351-358, https://doi.org/10.1016/0003-2697(79)90738-3.

37. Ellman, G.L. Tissue sulfhydryl groups. Archives of biochemistry and biophysics 1959, 82, 70-77, https://doi.org/10.1016/0003-9861(59)90090-6.

38. Paglia, D.E.; Valentine, W.N. Studies on the quantitative and qualitative characterization of erythrocyte glutathione peroxidase. The Journal of laboratory and clinical medicine 1967, 70, 158-169.

39. Bradley, P.P.; Priebat, D.A.; Christensen, R.D.; Rothstein, G. Measurement of cutaneous inflammation: estimation of neutrophil content with an enzyme marker. J Invest Dermatol 1982, 78, 206-209, https://doi.org/10.1111/1523-1747.ep12506462.

40. Aziza, S.; Aal, S.; Ismail, H. Effect of tramadol drug on some biochemical and immunological parameters in albino male rats; evaluation of possible reversal following its withdrawal. Benha Veterinary Medical Journal 2017, 33, https://doi.org/10.21608/bvmj.2017.30589.

41. Sastry, P.S.; Rao, K.S. Apoptosis and the nervous system. $J$ Neurochem 2000, 74, 1-20, https://doi.org/10.1046/j.1471-4159.2000.0740001.x.

42. Awadalla, E.A.; Salah-Eldin, A.-E. Molecular and histological changes in cerebral cortex and lung tissues under the effect of tramadol treatment. Biomedicine \& Pharmacotherapy 2016, 82, 269-280, https://doi.org/10.1016/j.biopha.2016.04.024.

43. Shaibah, H.S.; Elsify, A.K.; Medhat, T.M.; Rezk, H.M.; El-Sherbiny, M. Histopathological and immunohistochemical study of the protective effect of triptorelin on the neurocytes of the hippocampus and the cerebral cortex of male albino rats after short-term exposure to cyclophosphamide. J Microsc Ultrastruct 2016, 4, 123-132.

44. Hotta, H.; Uchida, S.; Kagitani, F. Effects of stimulating the nucleus basalis of Meynert on blood flow and delayed neuronal death following transient ischemia in the rat cerebral cortex. The Japanese journal of physiology 2002, 52, 383-393, https://doi.org/10.2170/jjphysiol.52.383.

45. Mattson, M.P. Neuroprotective signaling and the aging brain: take away my food and let me run. Brain research 2000, 886, 47-53, https://doi.org/10.1016/S0006-8993(00)02790-6.

46. Ahmadi, M.; Hoseinzade, A.; Haghighi, S.; Yosefi, S. Complex Partial Seizure and Hippocampus Atrophy Caused by Tramadol Abuse: A Case Study; 2017; In Press, https://doi.org/10.5812/amh.61240.

47. Paul, S.; Kulal, R.; Nath, R. Comparative evaluation of expression of VEGF(vascular endothelial growth factors) in periodontal tissues of healthy,chronic and aggressive periodontitis patients: an immunohistochemical study Letters in Applied NanoBioScience 2020, 9, 784-788, https://doi.org/10.33263/LIANBS91.784788.

48. Khodeary, M.F.; El-Din, A.A.S.; El Kholy, S.M. A histopathological and immunohistochemical study of adult rats'brain after long-term exposure to amadol (tramadol hydrochloride). Mansoura J Forensic Med Clin Toxicol 2010, 18, 1-24, https://doi.org/10.21608/MJFMCT.2010.53312.

49. Hassanzadeh, K.; Habibi-asl, B.; Farajnia, S.; Roshangar, L. Minocycline prevents morphine-induced apoptosis in rat cerebral cortex and lumbar spinal cord: a possible mechanism for attenuating morphine tolerance. Neurotoxicity research 2011, 19, 649-659, https://doi.org/10.1007/s12640-010-9212-0.

50. Sim, Y.J.; Kim, S.S.; Kim, J.Y.; Shin, M.S.; Kim, C.J. Treadmill exercise improves short-term memory by suppressing ischemia-induced apoptosis of neuronal cells in gerbils. Neuroscience letters 2004, 372, 256261, https://doi.org/10.1016/j.neulet.2004.09.060.

51. Ismail, M.; Al-Naqeep, G.; Chan, K.W. Nigella sativa thymoquinone-rich fraction greatly improves plasma antioxidant capacity and expression of antioxidant genes in hypercholesterolemic rats. Free radical biology \& medicine 2010, 48, 664-672, https://doi.org/10.1016/j.freeradbiomed.2009.12.002.

52. Al-Brakati, A.Y.; Fouda, M.S.; Tharwat, A.M.; Elmahallawy, E.K.; Kassab, R.B.; Abdel Moneim, A.E. The protective efficacy of soursop fruit extract against hepatic injury associated with acetaminophen exposure is mediated through antioxidant, anti-inflammatory, and anti-apoptotic activities. Environmental science and pollution research international 2019, 26, 13539-13550, https://doi.org/10.1007/s11356-019-04935-3.

53. Yin, M.; Jiang, N.; Guo, L.; Ni, Z.; Al-Brakati, A.Y.; Othman, M.S.; Abdel Moneim, A.E.; Kassab, R.B. Oleuropein suppresses oxidative, inflammatory, and apoptotic responses following glycerol-induced acute kidney injury in rats. Life sciences 2019, 232, https://doi.org/10.1016/j.lfs.2019.116634.

54. Yu, G.; Liang, Y.; Zheng, S.; Zhang, H. Inhibition of Myeloperoxidase by N-Acetyl Lysyltyrosylcysteine Amide Reduces Oxidative Stress-Mediated Inflammation, Neuronal Damage, and Neural Stem Cell Injury 
in a Murine Model of Stroke. The Journal of pharmacology and experimental therapeutics 2018, 364, 311322, https://doi.org/10.1124/jpet.117.245688.

55. Yan Kazakov; Aleksey Tarasov; Lyudmila Alyoshina; Brainina, K. Interplay between antioxidant activity, health and disease. Biointerface Research in Applied Chemistry 2019, 10, 4893 - 4901 https://doi.org/10.33263/BRIAC101.893901.

56. Sureda, A.; Ferrer, M.D.; Tauler, P.; Maestre, I.; Aguilo, A.; Cordova, A.; Tur, J.A.; Roche, E.; Pons, A. Intense physical activity enhances neutrophil antioxidant enzyme gene expression. Immunocytochemistry evidence for catalase secretion. Free radical research 2007, 41, 874-883, https://doi.org/10.1080/10715760701416459. 